International Journal of Wireless \& Mobile Networks (IJWMN) Vol. 3, No. 2, April 2011

\title{
Multi-Criteria Vertical Handover for Heterogonous Networks
}

\author{
Mohammed M. Alkhawlani \\ Faculty of Science and Engineering, University of Science and Technology, Sana'a, \\ Yemen \\ m. alshadadidust.edu.ye
}

\begin{abstract}
In the next generation heterogeneous wireless networks, a user with a multi-interface terminal may have network access from different service providers using various technologies. Vertical Handover (VHO) is the capability to switch on-going connections from one Radio Access Network (RAN) to another. This switching is based on the discovered accesses, QoS constraints, operator policies, user preferences and available system capacity and utilization. Optimizing the VHO process is an important issue of research, which leads to reduction of network signaling and mobile device power loss and on the other hand improves network quality of service (QoS) and grade of service (GoS).

In this paper, a decision support system is developed to address the VHO problem. This system combines fuzzy logic and TOPSIS, a MCDM algorithm, to the problem of VHO. This combination decreases the influence of the dissimilar, imprecise, and contradictory measurements for the VHO criteria coming from different sources. A performance analysis is done and results are compared with traditional algorithms for $V H O$. These results demonstrate a significant improvement with our developed algorithm.
\end{abstract}

\section{KEYWORDS}

Vertical Handover, Heterogeneous Networks, Fuzzy Logic, MCDM, TOPSIS

\section{INTRODUCTION}

The future Heterogeneous Wireless Network (HWN) is composed of multiple Radio Access Technologies (RATs) and domains, therefore, new Radio Resource Management (RRM) schemes and mechanisms are necessary to benefit from the individual characteristics of each RAT and to exploit the gain resulting from jointly considering the whole set of the available radio resources in each RAT. These new RRM schemes have to support mobile users who can access more than one RAT alternatively or simultaneously using a multi-mode terminal.

An important RRM consideration for overall HWN stability, resource utilization, user satisfaction, and Quality of Service (QoS) provisioning is the selection of the most optimal and promising Access Network (AN) for a new service request or a handoff request. However, choosing the best RAT is not a trivial task and there are many parameters, criteria, and viewpoints to take into account when selecting the best AN. The RRM mechanism that is responsible for selecting the most optimal and promising AN for a handoff service request in the HWN is called the Vertical Handover (VHO).

While the horizontal handover takes place between points of attachment in the same RAT (for example, between two neighboring base stations of a cellular network), the VHO occurs between points of attachment supporting different RATs (for example, between an IEEE 802.11 access point and a cellular network base station). VHO (also called intersystem handover) enables users to access several networks such as WLAN, WMAN, WPAN, and WWAN in parallel. It allows the applications even the real time application to be seamlessly transferred among different networks. In order to achieve seamless vertical handover in heterogeneous 
network environments, it is necessary to guarantee service continuity and QoS, which means low latency and low packet loss during handover.

[1] presents an FL based IP-centric vertical handoff decision algorithm and execution scheme between a WWAN and WLAN. In [2], [3], a cost based function that effects the characteristics of different networks is used in the handover decision algorithms and a network elimination factor is introduced to exclude those networks that cannot meet the QoS constraints for specific services. Several VHO schemes are proposed in [4], [5] trying to reduce the latency, packet loss and generally optimize the handover procedure. A segment selection algorithm based on the fuzzy multiple objective decision making system is presented by P. Chan et al. [6]. [7] has described adaptation of ELECTRE, MCDM tool, for ranking network alternatives during the network selection process. Q. Song and A. Jamalipour in [8] propose the combined application of two mathematical techniques in an algorithm for network selection between UMTS and WLAN, where the Analytic Hierarchy Process (AHP) and the Grey system theory are used to evaluate the users preferences and service requirements, and combine the priority settings of the QoS attributes with the performances of the network alternatives to make the network selection decision. TOPSIS, MCDM tool, is applied to the problem of network selection [9]. The proposed algorithm depends upon the QoS requirements of the service being requested by the user device. J. Noonan et al. in [10] examine the VHO decision, and propose that the selection decision is made by the client application by considering network characteristics and cost. Venom et al. [11] propose a user-centric selection approach that estimates user satisfaction regarding the selection of radio links in heterogeneous wireless networks. [12] proposes a net utility-based network selection algorithm, where a utility function is used to reflect the user satisfaction level to QoS and a cost function is used to reflect the cost for service. H. Jia et al. in [13] propose a low complexity, centralized network-controlled selection scheme, aiming to optimally distribute the end users to the networks of the heterogeneous wireless system to maximize the global spectrum efficiency. A dynamic user-centric network selection and decision process which optimizes handover across heterogeneous networks is proposed in [14]. In [15] A. Iera et al. present a multi-criteria network selection algorithm that relies on a suitably defined cost function, which takes into account metrics reflecting both network related and user preference related objectives. In [16], G. Koundourakis et al. introduce an operator-centric approach for access and interface selection (AIS) in a co-existed UMTS, WLAN and DVB-T heterogeneous wireless environment. The proposed approach focuses on the optimization of the resource utilization, while ensuring acceptable QoS provision to the end users.

The main contributions of this paper can be summarized as follows

- The development of a new class of VHO algorithms that are based on hybrid parallel Fuzzy Logic (FL) based decision and TOPSIS MCDM systems. This helps out to achieve adaptive, flexible, and scalable VHO algorithms.

- The FL based solution has been thought to be a good candidate for reaching suitable VHO decisions from such imprecise and dissimilar information.

- The FL based VHO solution is able to response to the changing conditions of the NGWN environments and the accumulated experience of the operators and users.

- FL based solution is easy to modify by tuning and adjusting the inference rules and membership functions.

- The idea of the parallel FLC reduces the number and complexity of the inference rules used in the FL based solution, which helps out in achieving more scalable solutions. 
- In a very complex and uncertain environments such as NGWN, MCDM can sufficiently reduce the uncertainty and doubt about the alternatives and allows a reasonable choice to be made from among them.

- VHO problem is a multi criteria problem in nature and the flexibility and complementary VHO multi-criteria can be utilized to provide a solution that can cope with the different viewpoints and goals.

The most important related work of the VHO problem is presented in the current Section. A brief overview for FLC and TOPSIS is presented in section 2. An VHO algorithm for coexisted WWAN, WMAN and WLAN environment is proposed in Section 3. The simulation models and performance metrics are presented in Section 4. The performance evaluation of the proposed

algorithm is carried out in Section 5. The conclusions and future works are presented in Section 6.

\section{TOPSIS AND FLC}

TOPSIS (Technique for Order Preference by Similarity to Ideal Solution) is a widely used MADM algorithm developed by Yoon and Hwang [17]. It is applicable for problem spaces that have the attributes with monotonically increasing or decreasing levels of utility. The algorithm calculates perceived positive and negative ideal solutions based on the range of attribute values available for the alternatives. The premise of the algorithm is that the best solution is the one with the shortest distance to the positive ideal solution and longest distance from the negative ideal solution, where distances are measured in Euclidean terms.

Let's assume that we have two criteria $X_{1}$ and $X_{2}$ as instance. $h_{+}$is the ideal solution and $h_{-}$is the negative solution. $A l$ and $A 2$ are different alternatives; $1 S_{+}$and $1 S_{-}$represent the distance between $A l$ and $h+$ and $h_{-}$respectively.

$2 S_{+}$and $2 S_{-}$represent the distance between $A 2$ and $h+$ and $h_{-}$respectively. If the relative distance between the ideal solution $h+$ and $A_{l}$ is shorter than $A_{2}$, then, the ranking of $A_{l}$ is more preferred than $A$ 2. In general, the overall calculative procedures of TOPSIS are as following:

1) Establish the normalized decision matrix.

2) Determine the ideal solution and negative ideal solution.

3) Calculate the distance from the ideal and negative ideal solution for each alternative.

4) Calculate the relative closeness to the ideal solution for each alternative.

5) Rank the preference order.

Fuzzy Logic Control (FLC) is a non-linear control method, which attempts to apply the expert knowledge of an experienced user to the design of a controller. The fuzzy control system contains four main parts, the fuzzifier, the fuzzy rules base, the fuzzy inference engine, and the defuzzifier. The fuzzifier maps the real valued numbers into a fuzzy set, which is the input to the fuzzy inference engine. The fuzzifiction process includes the definition of the universe of discourse and the specification of the linguistic variables, the fuzzy sets for the linguistic variables, and the membership functions for the specified fuzzy sets. The fuzzy rules base consists of a collection of fuzzy IF-THEN rules to represent the human knowledge about the problem. The fuzzy inference engine maps the input fuzzy sets into output fuzzy sets and 
handles the way in which the rules are combined just as humans use many different types of inferential procedures. The defuzzifier task is the reverse operation to the fuzzifier. It maps the output fuzzy sets into real valued numbers.

\section{MULTI-CRITERIA VHO SOLUTION}

In order to formulate the VHO as MCDM problem, we consider $A=\{A i$, for $i=1,2, \ldots, n\}$, a set of finite number of alternatives. Also, we consider $C=\left\{C_{j}\right.$, for $\left.j=1,2, \ldots, m\right\}$ to be a set of attributes against which the alternatives have to be judged and $w 1, w 2, \ldots, w m$ to represent the relative importance of these attributes. For the vertical handover problem, the following is a representative set of criteria that are considered in the decision making process using TOPSIS.

1) Resource Availability (RA): to avoid any network congestion and to keep a balanced load between the coexisting networks, the new or handoff calls are usually connected to the network with higher available resources.

2) Received Signal Strength (RSS): it is usually better to connect the user to the network with the strongest received signal, because weak received signal can cause unnecessary handover, call drop, and packets or bits errors.

3) Mobile Station Speed (MSS): to avoid unnecessary handover overhead when moving from the ANs with small coverage area such as WPAN or WLAN to ANs with larger coverage area such as WMAN and WWAN, the low speed users are usually connected to the ANs with small coverage area and the high speed users are connected to the ANs with large coverage area.

4) Service Types (ST): due to the different QoS architectures and schemes used by the different networks, some networks such as WLAN are preferred for data, bursty services, and streaming multimedia services and other networks such as $3 \mathrm{G}$ networks are preferred for voice and conversational multimedia services.

5) User Preferred Price (UPP): the operators assign the links of high cost networks (with better QoS) for users who are willing to pay more and the links of low cost networks to other users.

6) Security (SEC): for some applications, confidentiality or integrity of the transmitted data can be critical. For this reason, a network with higher security level may be chosen over another one which would provide lower level of data security.

\subsection{The Parallel FL Component}

The measurements for the criteria mentioned above are in general very dissimilar, imprecise, contradictory, and coming from different sources. For example, the ranges of RSS and RA values are different in each type of RATs and the same value in every range has different performance scores. In addition, VHO solution has to be able to response to the changing conditions of the NGWN environments and the accumulated experience of the operators and users. The only way for TOPSIS to do so is to change the criteria weights manually to get better total performance. To decrease the influence of the above obstacles, several parallel FLC subsystems are used. Each subsystem considers one of the important VHO criteria mentioned above. The RA subsystem considers the resource availability criterion. The RSS subsystem considers the received signal strength criterion. The MSS subsystem considers the mobile station speed criterion. The ST subsystem considers the service type criterion. The UPP 
subsystem considers the user preferred price criterion. The SEC subsystem considers the security criterion. The MSS subsystem is described in the following paragraph as an example.

Figure 1 shows the MSS FL system. MSS system has only one input variable "MSS" to describe the mobile station speed. The universe of discourse is selected to represent the expected speed of walking user. The universe starts with $0 \mathrm{~km} / \mathrm{hr}$ to represent the stationary user and ends with the $10 \mathrm{~km} / \mathrm{hr}$ to represent the running user. Three linguistic variables have been used to describe the universe of discourse \{Low, Medium, and High\}. Figure 2 shows the membership functions of the "MSS" variable. The significant overlapping between the first and second membership function and between the second and third membership function implies smoother and easier to implement control surface for output variables. In addition, it achieves robust inference since in the overlapped area at least two rules are usually applied. The MSS FL based system has three inference rules as shown in table 1. Table 1 shows that the input variable MSS has three membership functions Low (L), Medium (M), and High (H) and every output variable has four membership functions TR (Totally Reject), PR (Probability Reject), PA (Probability Accept), and TA (Totally Accept). The inference rules of the MSS FL based system are designed with the aim of minimizing the handoff rate in mind and consequently utilize the networks' resources in more efficient way. The slow users are attached to the small coverage area ANs and the fast users to the larger coverage area.

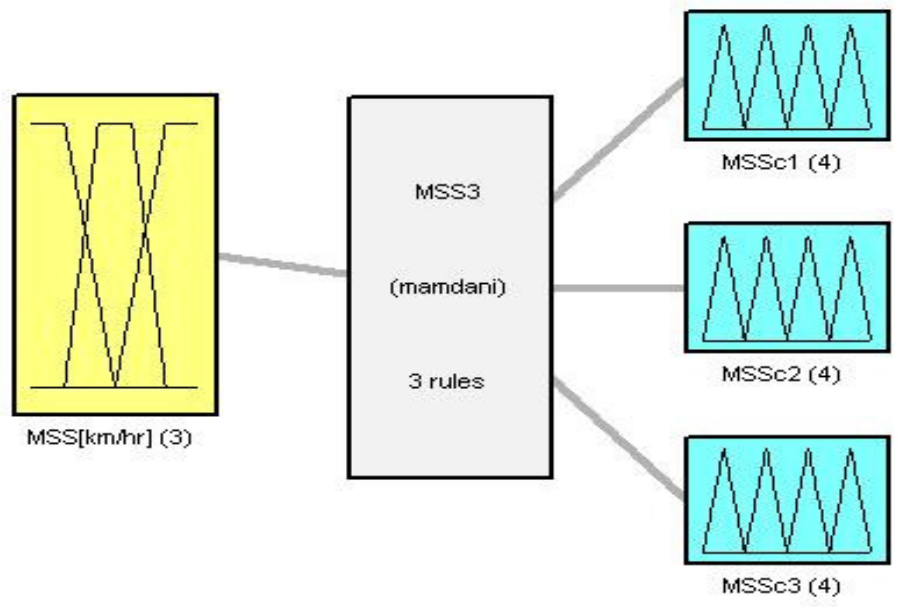

System MSS3: 1 inputs, 3 outputs, 3 rules

Figure 1: The MSS FL system

Table 1: The inference rules of the MSS FL based system

\begin{tabular}{|c|c|c|c|c|}
\hline Rule No. & $M S S$ & $M S S_{c 1}$ & $M S S_{c 2}$ & $M S S_{c 3}$ \\
\hline 1 & L & PR & TA & PR \\
\hline 2 & M & PA & PR & TA \\
\hline 3 & H & TA & TR & PR \\
\hline
\end{tabular}




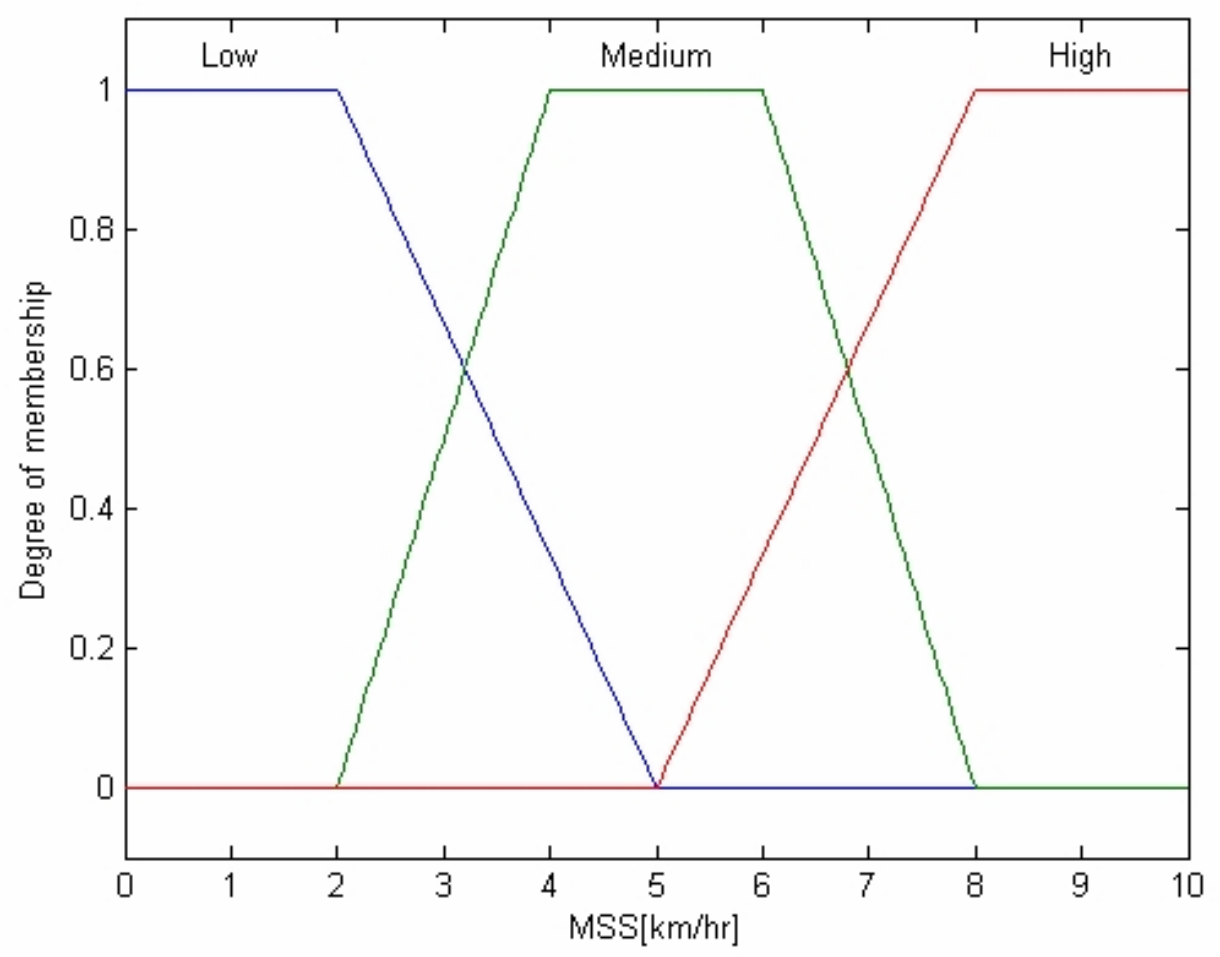

Figure 2: Membership functions of "MSS" input variable

\subsection{The MCDM Component}

There are three alternatives for the MCDM, the first one is a WWAN network, the second is a WMAN network, and the third one is a WLAN network. The input criteria of the MCDM are the outputs of the FL based control subsystems.

Since all the outputs of FL subsystems are in the range [0,1], there is not any need to scale the criteria performance against alternatives. The decision problem can be concisely expressed in the normalized decision matrix shown in equation 1.

$$
N D M=\left(\begin{array}{ccc}
\boldsymbol{R A} A_{C 1} & \boldsymbol{R A}_{C 2} & \boldsymbol{R A}_{C 3} \\
\boldsymbol{R S S} S_{C 1} & \boldsymbol{R S S _ { C 2 }} & \boldsymbol{R S S}_{C 3} \\
\boldsymbol{M S S _ { C 1 }} & \boldsymbol{M S S _ { C 2 }} & \boldsymbol{M S S _ { C 3 }} \\
\boldsymbol{S T _ { C 1 }} & \boldsymbol{S T _ { C 2 }} & \boldsymbol{S T _ { C 3 }} \\
\mathbf{U P P _ { C 1 }} & \mathbf{U P P _ { C 2 }} & U \boldsymbol{U P P _ { C 3 }} \\
\boldsymbol{S E C _ { C 1 }} & \mathbf{S E C _ { C 2 }} & \mathbf{S E C _ { C 3 }}
\end{array}\right)
$$

The next step is to decide on the relative importance of each of the attributes involved in the decision about network selection. The criteria with more importance to the operator and user can be assigned higher weight. For this purpose, each of the attribute is assigned a specific weight, such that 


$$
T W=W_{r a}+W_{r s s}+W_{m s s}+W_{s t}+W_{u p p}+W_{s e c}=1
$$

where $W_{r a}$ is the assigned weight for the resource availability criterion. $W_{r s s}$ is the assigned weight for the received signal strength criterion. Wmss is the assigned weight for the mobile station speed criterion. $W_{s t}$ is the assigned weight for the service type criterion. $W_{u p p}$ is the assigned weight for the user preferred price criterion. $W_{s e c}$ is the assigned weight for the security criterion. $T W$ is the total weight and is calculated using 2 . Using these assigned weights, the matrix in equation 1 is updated as shown in 3.

$W D M=\left(\begin{array}{ccc}W_{r a} * R A_{C 1} & W_{r a} * R A_{C 2} & W_{r a} * R A_{C 3} \\ W_{r s s} * R S S_{C 1} & W_{r s s} * R S S_{C 2} & W_{r s s} * R S S_{C S} \\ W_{m s g} * M S S_{C 1} & W_{m s s} * M S S_{C 2} & W_{r m s s} * M S S_{C 3} \\ W_{s t} * S T_{C 1} & W_{s t} * S T_{C 2} & W_{s t} * S T_{C 3} \\ W_{\text {wp }} * U P P_{C 1} & W_{\text {upp }} * U P P_{C 2} & W_{\text {upp }} * U P P_{C 3} \\ W_{s e c} * S E C_{C 1} & W_{s e c} * S E C_{C 2} & W_{s e c} * S E C_{C 3}\end{array}\right)$

The next step is to find the best and worst value for each of the attribute. Depending on the attribute, the best (or the worst) value can be either the maximum or the minimum value. For example, in the case of attribute for received signal strength, the best value will be the highest and worst value will be the lowest. For each of the alternatives under consideration (WWAN, WMAN, and WLAN), the measure of separation, both for the best and worth cases, is calculated as shown in equations 4 and 5 respectively.

$S_{\text {bath }}$

$=\sqrt{\left(R A_{C i}-R A_{b a t}\right)^{2}+\left(R S S_{a i}-R S S_{b a t}\right)^{2}+\left(M S S_{a i}-M S S_{b a s t}\right)^{2}+\left(S T_{a i}-S T_{b a t}\right)^{2}+\left(U P P_{a i}-U P P_{b a t}\right)^{2}+\left(S E C_{a i}-S E C_{b a t}\right)^{2}}$

$S_{\text {Hurst }}$

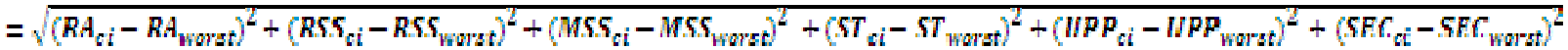

The preference order for each alternative $P i$, measured in terms of distances $S$ from the best and worst solutions, is represented by the following formulation

$$
P_{i}=\frac{S_{\text {worst }}^{i}}{S_{\text {worgt }}^{t}+S_{\text {best }}^{i}}
$$

\section{THE PERFORMANCE EVALUATION}

Our proposed solution is evaluated using the simulation approach. This section presents the used performance metrics and simulation models.

\subsection{The performance metrics}

In this paper four performance evaluation metrics have been used to evaluate our algorithms. The used metrics can be described briefly as follows. 
International Journal of Wireless \& Mobile Networks (IJWMN) Vol. 3, No. 2, April 2011

- Handover rate $\left(P_{n}\right)$ : reducing the number of handovers is usually preferred as frequent handovers would cause wastage of network resources. $P_{n}$ can be calculated as shown in equation 7.

$$
P_{n}=\frac{\text { Number of Hundoff Requesls }}{\text { Total Number of Users }}
$$

- Handover failure rate $\left(P_{f}\right)$ : a handover failure occurs when the handover is initiated but the target network does not have sufficient resources to complete it, or when the mobile terminal moves out of the coverage of the target network before the process is analyzed. $P_{f}$ can be calculated as shown in equation 8.

$$
P_{f}=\frac{\text { Number of Unsuccessful Handoff of Requests }}{\text { Number of Haruloff Requests }}
$$

- The percentage of users who are assigned to networks of their preference $(P u)$ : this metric reflects the user point of view about the performance of the selection process.

- The usage percentage of the low cost network resources (i.e., WLAN) $\left(P_{o}\right)$ : this metric reflect the operator point of view because it utilizes the resources of the high cost networks (i.e., WMAN and WWAN). Simply, $P_{o}$ can be calculated as the percentage between the number of users in WLAN and the total number of users as shown in equation 9.

$$
P_{o}=\frac{\text { Number of users in WLAN }}{\text { I'otal Number of Users }}
$$

\subsection{The simulation environment}

A modified version of MATLAB based simulator called RUNE [18], [19] has been used. The simulation environment defines a system model, a mobility model, a propagation model, and services model. The system model considers the coexistence of three types of wireless access networks.

The first network is a CDMA based WWAN with seven macro cells and cell radius of $1000 \mathrm{~m}$. The second one is a CDMA based WMAN with twelve macro cells and cell radius of $500 \mathrm{~m}$. The third one is a CDMA based WLAN with eighty four micro cells and cell radius of $100 \mathrm{~m}$. All cells have standard hexagonal shapes with Omni-directional antennas.

The mobiles are randomly distributed over the system. In every slot each mobile is moved a random distance in a random direction at defined time steps. The movement pattern of each mobile depends on the velocity and acceleration. The velocity is a vector quantity with magnitude and direction. The velocity of the $i t h$ mobile is updated according to equation 10.

$$
V_{i}=V_{I-1} \cdot P+\sqrt{1-P^{2}} \cdot V_{m} \cdot X
$$


where $V_{i}$ is the complex speed [m/s]. $V_{i-1}$ is the complex speed in the previous time step. $X$ is a Rayleigh distributed magnitude with mean 1 and a random direction. $V_{m}$ is the mean speed of mobiles. $P$ is the correlation of the velocity between time steps. $\mathrm{P}$ depends on both amean which is the mean acceleration of the mobile user and $V_{\text {mean }} . V_{m}$ has been set to $15[\mathrm{~m} / \mathrm{s}]$ and the mean acceleration has been set to 1 .

The propagation model simulates the different losses and gains during the signal propagation between the transmitter and the receiver in the system environment. The wireless propagation model used in this paper is described in a logarithmic scale as in equation 11.

$$
G=G_{D}+G_{F}+G_{K}+G_{A}
$$

Equation 11 contains four components; the first component is the distance attenuation $G D$ that is calculated by Okumura- Hata formula presented in [20]. The second component is the shadow fading $G F$ that is modeled as a log-normal distribution with standard deviation of $6 \mathrm{~dB}$ and $0 \mathrm{~dB}$ mean. The third component is the Rayleigh fading GR that is modeled using a Rayleigh distribution. The forth component is the antenna gain $G_{A}$ that adds the antenna gain in $\mathrm{dB}$.

Adaptive service model is considered in our simulation. The service $i$ is mainly characterized by its bit rate requirement "RateReqc" and delay requirement "DelayReqc". The users are generated according to Poisson process. The service holding time is exponential distribution with mean holding time equals to 120 seconds.

\section{THE RESULTS STUDY}

Three different reference algorithms are simulated and evaluated against our developed solution. The first algorithm is a terminal speed based VHO where high speed users are sent to the highcoverage network and the low and medium speed users are sent to the smaller coverage area networks. The second algorithm is a resource availability based VHO where the users are assigned to the network with higher resources. The third algorithm is based on a received signal VHO where the users are assigned to the network with higher signal strength. Some simulation results for different sets of users are presented in this section. From both Figure 3 and the numerical samples for $P_{n}$ values shown in Table 2, the reduction in the number of handovers in our solution can be seen. For example, with 1234 users in the environment, the handover rate with the terminal-speed based algorithm is $23.9 \%, 34.8 \%$ with the resource availability based algorithm, and $26.4 \%$ with the signal strength based algorithm. The same number with the combined FL and TOPSIS algorithm is around 19.7\%. In general, our developed FL-TOPSIS solution achieves around 3\% enhancement over the terminal speed based algorithm and around $7 \%$ and $4 \%$ over the resource availability based algorithm and the signal strength based algorithm respectively. 
International Journal of Wireless \& Mobile Networks (IJWMN) Vol. 3, No. 2, April 2011

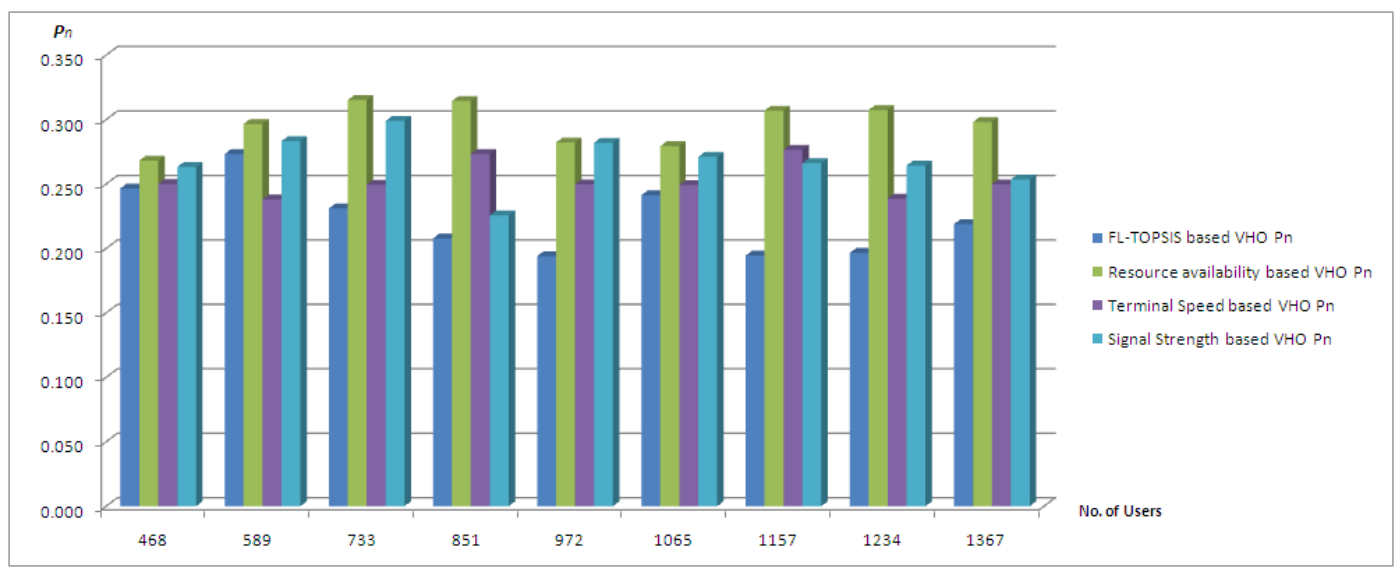

Figure 3: $P_{n}$ values of the combined FL and TOPSIS based algorithm against the reference algorithms

Table 2: $P_{n}$ values of the combined FL and TOPSIS based algorithm against the reference algorithms

\begin{tabular}{|c|c|c|c|c|}
\hline No. of Users & $\begin{array}{c}\text { FL-TOPSIS } \\
\text { VHO } P_{n}\end{array}$ & $\begin{array}{c}\text { MSS } \\
\text { VHO } P_{n}\end{array}$ & $\begin{array}{c}\text { RA } \\
\text { VHO } P_{n}\end{array}$ & $\begin{array}{c}\text { RSS } \\
\text { VHO } P_{n}\end{array}$ \\
\hline 468 & 0.247 & 0.25 & 0.268 & 0.263 \\
\hline 589 & 0.273 & 0.238 & 0.297 & 0.283 \\
\hline 733 & 0.231 & 0.250 & 0.315 & 0.299 \\
\hline 851 & 0.208 & 0.273 & 0.314 & 0.226 \\
\hline 972 & 0.194 & 0.250 & 0.282 & 0.282 \\
\hline 1065 & 0.241 & 0.249 & 0.279 & 0.271 \\
\hline 1157 & 0.194 & 0.276 & 0.307 & 0.266 \\
\hline 1234 & 0.197 & 0.239 & 0.308 & 0.264 \\
\hline 1367 & 0.219 & 0.250 & 0.298 & 0.253 \\
\hline
\end{tabular}

From both Figure 4 and the numerical samples for Pf values shown in Table 3, the enhancement in the handover failure rate in our solution can be seen. For example, with 1234 users in the environment, the handover failure rate with the terminal-speed based algorithm is $23.6 \%, 24.4 \%$ with the resource availability based algorithm, and $17.3 \%$ with the signal strength based algorithm. The same number with the combined FL and TOPSIS algorithm is around $18 \%$. In general, our developed FL-TOPSIS solution achieves around 7\% enhancement over the terminal speed based algorithm and around $8 \%$ and $0.5 \%$ over the resource availability based algorithm and the signal strength based algorithm respectively. 
International Journal of Wireless \& Mobile Networks (IJWMN) Vol. 3, No. 2, April 2011

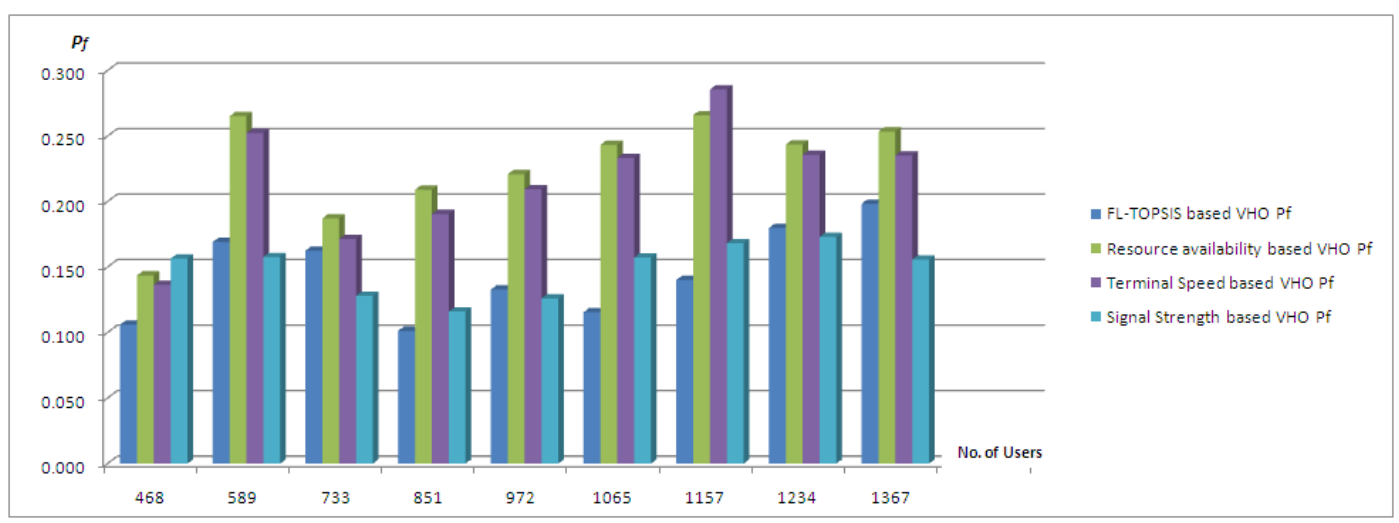

Figure 4: $P_{f}$ values of the combined FL and TOPSIS based algorithm against the reference algorithms

Table 3: $P_{f}$ values of the combined FL and TOPSIS based algorithm against the reference algorithms

\begin{tabular}{|c|c|c|c|c|}
\hline No. of Users & $\begin{array}{c}\text { FL-TOPSIS } \\
\text { VHO } P f\end{array}$ & $\begin{array}{c}\text { MSS } \\
\text { VHO } P_{f}\end{array}$ & $\begin{array}{c}\text { RA } \\
\text { VHO } P_{f}\end{array}$ & $\begin{array}{c}\text { RSS } \\
\text { VHO } P_{f}\end{array}$ \\
\hline 468 & 0.106 & 0.136 & 0.144 & 0.157 \\
\hline 589 & 0.169 & 0.252 & 0.265 & 0.158 \\
\hline 733 & 0.163 & 0.172 & 0.187 & 0.128 \\
\hline 851 & 0.101 & 0.191 & 0.209 & 0.116 \\
\hline 972 & 0.133 & 0.209 & 0.221 & 0.126 \\
\hline 1065 & 0.116 & 0.233 & 0.243 & 0.157 \\
\hline 1157 & 0.140 & 0.286 & 0.266 & 0.168 \\
\hline 1234 & 0.180 & 0.236 & 0.244 & 0.173 \\
\hline 1367 & 0.198 & 0.235 & 0.254 & 0.156 \\
\hline
\end{tabular}

From both Figure 5 and the numerical samples for Po values shown in Table 4, the great improvement in the percentage of the users who are assigned to low cost networks (i.e. WLAN) in our solution can be seen. For example, with 1234 users in the environment, the percentage of satisfied users with the terminal-speed based algorithm is $36.4 \%, 28.5 \%$ with the resource availability based algorithm, and $35.4 \%$ with the signal strength based algorithm. The same number with the combined FL and TOPSIS algorithm is around 40.6\%. In general, our developed FL-TOPSIS solution achieves around 4\% enhancement over the terminal speed based algorithm and around $13 \%$ and $7 \%$ over the resource availability based algorithm and the signal strength based algorithm respectively. 
International Journal of Wireless \& Mobile Networks (IJWMN) Vol. 3, No. 2, April 2011

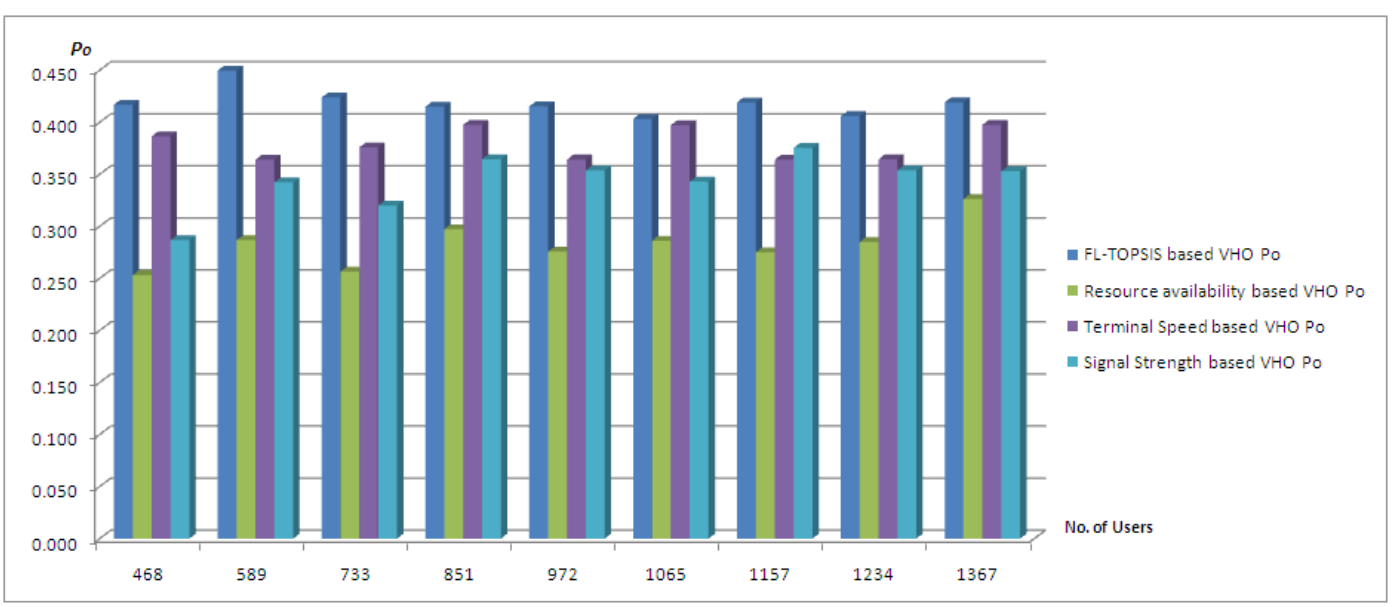

Figure 5: $P_{o}$ values of the combined FL and TOPSIS based algorithm against the reference algorithms

Table 4: $P_{o}$ values of the combined FL and TOPSIS based algorithm against the reference algorithms

\begin{tabular}{|c|c|c|c|c|}
\hline No. of Users & $\begin{array}{c}\text { FL-TOPSIS } \\
\text { VHO } P_{o}\end{array}$ & $\begin{array}{c}\text { MSS } \\
\text { VHO } P_{o}\end{array}$ & $\begin{array}{c}\text { RA } \\
\text { VHO } P_{o}\end{array}$ & $\begin{array}{c}\text { RSS } \\
\text { VHO } P_{o}\end{array}$ \\
\hline 468 & 0.417 & 0.387 & 0.253 & 0.287 \\
\hline 589 & 0.450 & 0.364 & 0.287 & 0.342 \\
\hline 733 & 0.424 & 0.376 & 0.257 & 0.320 \\
\hline 851 & 0.415 & 0.397 & 0.297 & 0.365 \\
\hline 972 & 0.415 & 0.364 & .276 & 0.354 \\
\hline 1065 & 0.419 & 0.397 & 0.286 & 0.343 \\
\hline 1157 & 0.397 & 0.364 & 0.275 & 0.375 \\
\hline 1234 & 0.406 & 0.364 & 0.285 & 0.354 \\
\hline 1367 & 0.419 & 0.398 & 0.326 & 0.353 \\
\hline
\end{tabular}

From both Figure 6 and the numerical samples for $\mathrm{Pu}$ values shown in Table 5, the great improvement in the percentage of the users who are assigned to the network of their preference in our solution can be seen. For example, with 1234 users in the environment, the percentage of satisfied users with the terminal-speed based algorithm is $34.8 \%, 38.5 \%$ with the resource availability based algorithm, and $35.3 \%$ with the signal strength based algorithm. The same number with the combined FL and TOPSIS algorithm is around 52.5\%. In general, our developed FL-TOPSIS solution achieves around 16\% enhancement over the terminal speed based algorithm and around $14 \%$ and $16 \%$ over the resource availability based algorithm and the signal strength based algorithm respectively. 
International Journal of Wireless \& Mobile Networks (IJWMN) Vol. 3, No. 2, April 2011

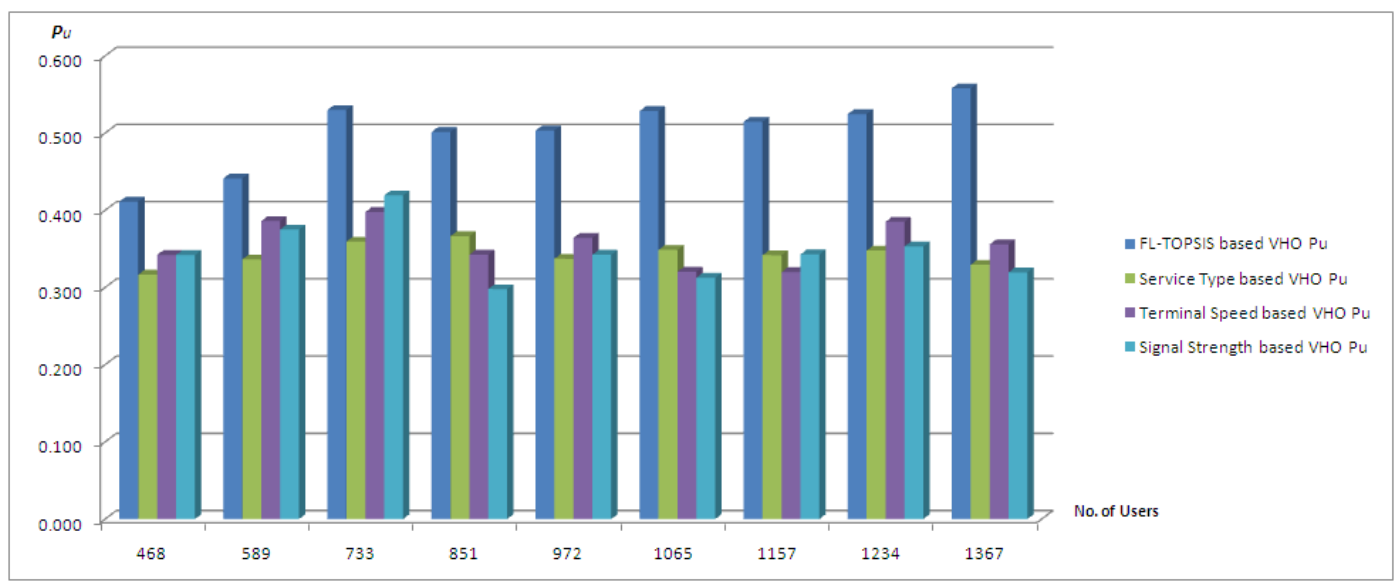

Figure 6: $P u$ values of the combined FL and TOPSIS based algorithm against the reference algorithms

Table $5 P u$ values of the combined FL and TOPSIS based algorithm against the reference algorithms

\begin{tabular}{|c|c|c|c|c|}
\hline No. of Users & $\begin{array}{c}\text { FL-TOPSIS } \\
\text { VHO } P u\end{array}$ & $\begin{array}{c}\text { MSS } \\
\text { VHO } P u\end{array}$ & $\begin{array}{c}\text { RA } \\
\text { VHO } P u\end{array}$ & $\begin{array}{c}\text { RSS } \\
\text { VHO } P u\end{array}$ \\
\hline 468 & 0.417 & 0.317 & 0.342 & 0.342 \\
\hline 589 & 0.441 & 0.336 & 0.386 & 0.375 \\
\hline 733 & 0.530 & 0.359 & 0.398 & 0.419 \\
\hline 851 & 0.501 & 0.366 & 0.342 & 0.298 \\
\hline 972 & 0.503 & 0.337 & 0.364 & 0.342 \\
\hline 1065 & 0.529 & 0.349 & 0.320 & 0.313 \\
\hline 1157 & 0.515 & 0.342 & 0.320 & 0.343 \\
\hline 1234 & 0.525 & 0.348 & 0.385 & 0.353 \\
\hline 1367 & 0558 & 0.329 & 0.356 & 0.320 \\
\hline
\end{tabular}

\section{CONCLUSIONS AND FUTURE WORK}

The paper proposes an artificial intelligent based operator algorithm for the VHO in co-existed WWAN, WMAN, and WLAN environment. The developed algorithm is based on the parallel FL decision and on the TOPSIS multiple criteria decision making tool. The algorithm can achieve scalable solution for the ANS and can be easily extended to deal with the increased number of RATs and criteria. This extension will not add an exponential complexity to the extended solution. The algorithm can easily response to the expected changes in the networks' conditions, users' preferences, or operator policies by tuning the fuzzy logic membership functions and rules or by tuning the TOPSIS weighs. The simulation results show that the proposed algorithm has a better and more robust performance over the several VHO reference algorithms. The developed solution is evaluated using simulation approaches. Its performance is compared against several reference algorithms. 
International Journal of Wireless \& Mobile Networks (IJWMN) Vol. 3, No. 2, April 2011

The simulation results show that the developed solution has a better and robust performance over the reference algorithm in terms of the handover rate number of successful handover, the operator benefits and the QoS.

Our future works can be extended in several directions. An optimum values for the weights of the different criteria can be found using a global optimization method. Also, the rules and membership functions of the fuzzy subsystems can be built or tuned using the genetic algorithms or the neural networks.

\section{REFERENCES}

[1] Y. Nkansah-Gyekye and J. I Agbinya, (2006) "Vertical handoff between WWAN and WLAN", IEEE International Conference on Networking, International Conference on Systems and International Conference on Mobile Communications and Learning Technologies (ICNICONSMCL06), pp 132

[2] F. Zhu and J. Mcnair, (2004) "Optimizations for vertical handoff decision algorithms", IEEE Wireless Communications and Networking Conference (WCNC 2004), Vol. 2, pp. 867872

[3] J. Mcnair and F. Zhu, (2004) "Vertical handoffs in fourth-generation multinetwork environments", IEEE Wireless Communication, Vol. 11, No. 3, pp. 815

[4] P. Cleyn, N. Wijngaert, L. Cerda , and C. Blondia,(2004) "A smooth handoff scheme using IEEE802.11 triggers - design and implementation”, Elsevier Computer Networks, Vol. 45, No. 3 , pp 345-361

[5] Y. Pan, M. Lee, J. Kim, and T. Suda, (2006) "An end-to-end multi-path smooth handoff scheme for stream Media”, IEEE Journal of Selected Areas of Communications, Vol. 22, No. 4, pp 653663

[6] P.M. Chan, Y.F. Hu, and R.E. Sheriff, (2002) "Implementation of fuzzy multiple objective decision making algorithm in a heterogeneous mobile environment," The IEEE Wireless Communications and Networking Conference, Vol.1, pp 332- 336

[7] F. Bari and V. Leung, (2007) "Application of ELECTRE to network selection in a heterogeneous wireless network environment," IEEE Wireless Communications and Networking Conference (WCNC 2007), pp 3810 - 3815

[8] Q. Song and A. Jamalipour, (2005) "Network selection in an integrated wireless LAN and UMTS environment using mathematical modeling and computing techniques," IEEE Wireless Communications, Vol. 12, No. 3, pp 4248

[9] F. Bari and V. Leung, (2007) "Multi-Attribute network selection by iterative TOPSIS for heterogeneous wireless access," IEEE Consumer Communications and Networking Conference (CCNC 2007), pp 808 - 812

[10] J. Noonan, P. Perry and J. Murphy, (2004) "Client controlled network selection," IEE International Conference on 3G Mobile Communication Technologies (3G 2004), pp 543 - 547

[11] E. Vanem, S. Svaet, and F. Paint, (2003) "Effects of multiple access alternatives in heterogeneous wireless networks", IEEE Wireless and Networking, Vol. 3, pp 1696-1700

[12] L. Liang, H. Wang, and P. Zhan, (2007) "Net utility-based network selection scheme in CDMA cellular/WLAN integrated networks,” IEEE Wireless Communications and Networking Conference (WCNC 2007), pp 3313 - 3317

[13] H. Jia, Z. Zhang, P. Cheng, H. Hwa Chen and S. Li, (2006) "Study on network selection for next-generation heterogeneous wireless networks," IEEE 17th International Symposium on Personal, Indoor and Mobile Radio Communications, pp 1 - 5 
International Journal of Wireless \& Mobile Networks (IJWMN) Vol. 3, No. 2, April 2011

[14] X. Cai, L. Chen, R. Sofia, and Y. Wu, (2007) "Dynamic and user-centric network selection in heterogeneous networks," IEEE International Performance, Computing, and Communications Conference (IPCCC 2007), pp 538 - 544

[15] A. Iera, A. Molinaro, C. Campolo, and M. Amadeo, (2006) "An access network selection algorithm dynamically adapted to user needs and preferences," IEEE 17th International Symposium on Personal, Indoor and Mobile Radio Communications, pp 1 - 5

[16] G. Koundourakis, D. I. Axiotis and M. Theologou, (2007) "Network-based access selection in composite radio environments", IEEE Wireless Communications and Networking Conference (WCNC 2007), pp 3877 - 3883

[17] C.L. Hwang and K. Yoon, (1981) "Multiple Attribute Decision Making: Methods and Applications", Springer Verlag

[18] J. Zander and S. Kim, (2001) Radio Resource Management for Wireless Networks, Artech House

[19] P. Bruin, RUNE 5, Report T/B 99:036, (1999) Ericsson Radio Systems AB

[20] M. Hata, (1980) "Empirical formula for propagation loss in land mobile radio service", IEEE Transaction on Vehicular Technology, pp 317-325

\section{Authors}

Mohammed M. Alkhawlani is a Senior Lecturer at the University of Science and Technology (UST), Sana'a, Yemen. He received his $\mathrm{PhD}$ in Data Communication and Networking Engineering from De Montfort University, UK, in July 2008. He received his MSc in Data Communication Systems from Brunel University, UK, in December 2003. He received his BSc in Computer Engineering from Cairo University, Egypt, in July 2001. His research interest is radio resource management in the next generation of wireless networks with the aid of artificial intelligence tools.

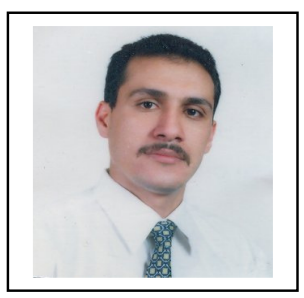

